Disorder and Fracture 


\section{Disorder and Fracture}

Edited by

J. C. Charmet and

S. Roux

Ecole Supérieure de Physique et Chimie Industrielles de Paris

Paris, France

and

E. Guyon

Université de Paris-Sud

Orsay, France 


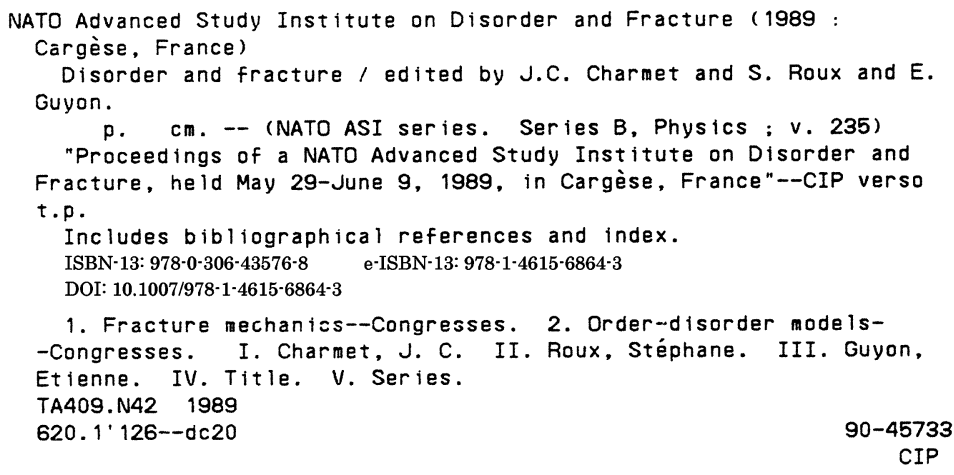

(C) 1990 Plenum Press, New York

A Division of Plenum Publishing Corporation 233 Spring Street, New York, N.Y. 10013

All rights reserved

No part of this book may be reproduced, stored in a retrieval system, or transmitted in any form or by any means, electronic, mechanical, photocopying, microfilming, recording, or otherwise, without written permission from the Publisher 


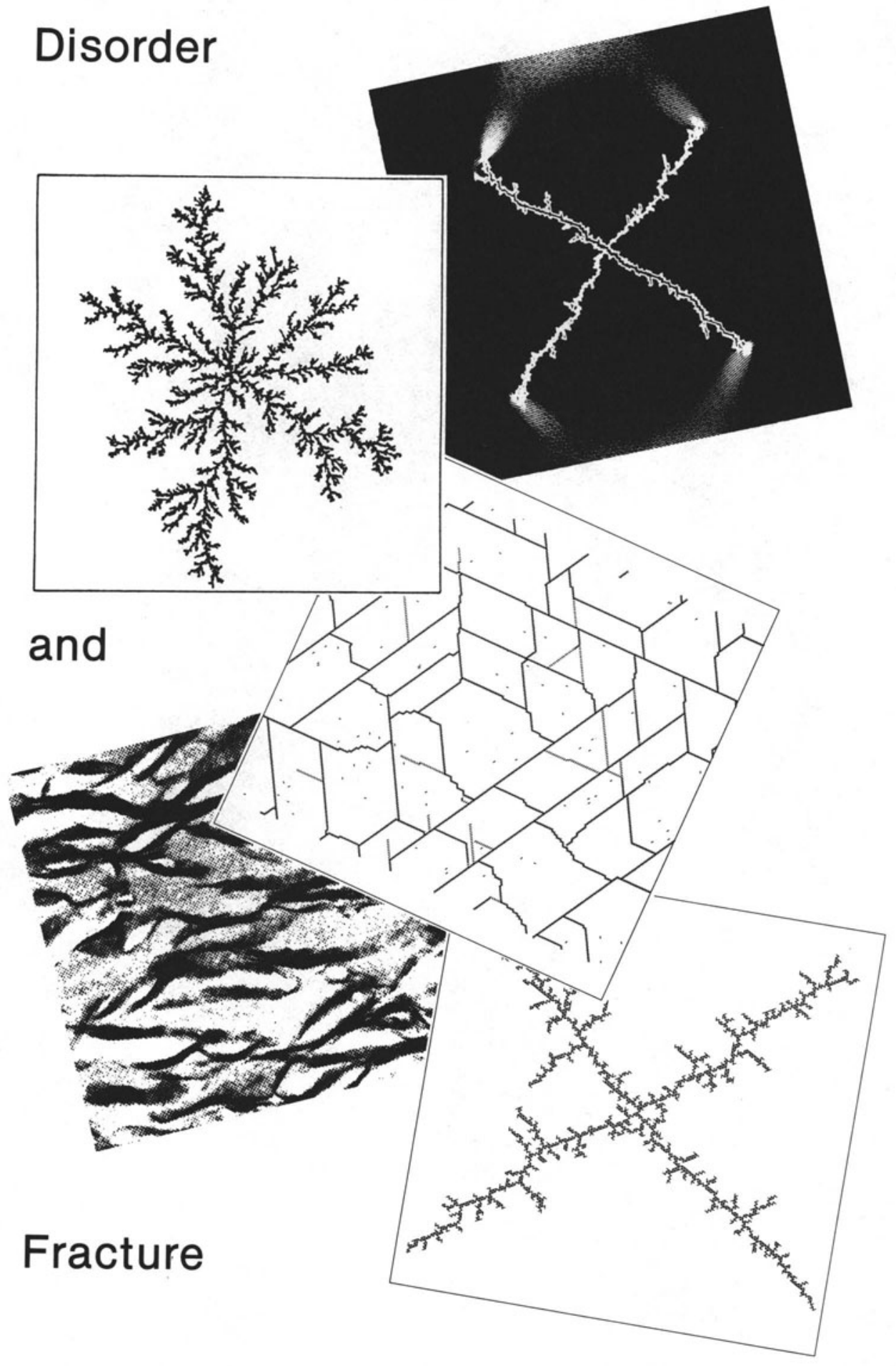




\section{Foreword}

Fracture, and particularly brittle fracture, is a good example of an instability. For a homogeneous solid, subjected to a uniform stress field, a crack may appear anywhere in the structure once the threshold stress is reached. However, once a crack has been nucleated in some place, further damage in the solid will in most cases propagate from the initial crack, and not somewhere else in the solid. In this sense fracture is an unstable process. This property makes the process extremely sensitive to any heterogeneity present in the medium, which selects the location of the first crack nucleated. In particular, fracture appears to be very sensitive to disorder, which can favor or impede local cracks. Therefore, in most realistic cases, a good description of fracture mechanics should include the effect of disorder. Recently this need has motivated work in this direction starting from the usual description of fracture mechanics.

Parallel with this first trend, statistical physics underwent a very important development in the description of disordered systems. In particular, let us mention the emergence of some "new" concepts (such as fractals, scaling laws, finite size effects, and so on) in this field. However, many models considered were rather simple and well adapted to theoretical or numerical introduction into a complex body of problems. An example of this can be found in percolation theory. This area is now rather well understood and accurately described. Nevertheless, examples of the exact application of this model to the real world are extremely scarce. Indeed, in most situations the geometric disorder present in a medium is not totally random, but rather generated through complex physical processes which introduce naturally correlations which are difficult to predict. Conversely, when heterogeneities are not correlated with each other, their concentration has very little likelihood of reaching a percolation threshold. But the very large effort devoted to the subject has permitted the elaboration of many concepts and tools which can certainly be used in much more complex cases. This is the reason why the physics of disorder is mature enough to be extended to more realistic cases, such as fracture of disordered solids, and indeed a few attempts have been made in this direction quite recently.

The conjunction of these two facts has motivated a natural coming together of both the mechanics and statistical physics communities on the problem of fracture and disorder, in order to share their concerns and their knowledge, their problems 
viii

and their tools. This was the genesis of the meeting held in Cargèse in June 1989, and this book gathers together the texts of the lectures given during this Institute.

Obviously this is only a first step along the path that we have to follow in order to begin to bring together the know-how and effort to attack the problems which we have to face in material science. Other meetings will have to be organized, common projects prepared, well-defined questions in a language understandable by both communities formulated, and so on. This renders both important and timely the need to prepare a good basis for common exchanges. This we have tried to achieve in this volume. It is naturally too early to try to guess what will result from such an approach, but it seems likely that many applications which combine instabilities and disorder will benefit from extensions of progress accomplished in fracture mechanics, and thus will be useful both in mechanics and in statistical mechanics.

These papers are grouped in sections covering "Tools", "Diffusion Limited Aggregation Model", "Statistical Models of Fracture", "Rheology and Fracture", "Materials and Applications", ranging from theoretical concepts to practical applications. Each section has a brief introduction which summarizes the links between different chapters which can be read independently.

We wish to thank the NATO Scientific Panel on nonlinear phenomena for their support of the Institute and this volume. We acknowledge also support from the CNRS, DRET and NSF. 


\section{Contents}

\section{Tools}

1 Fluctuations of Interfaces and Fronts 3

M. Kardar

2 Introduction to Multifractality

S. Roux and A. Hansen

3 Statistical Theory of Fragmentation

S. Redner

\section{Diffusion-Limited Aggregation Model}

4 Theory and Simulation of Diffusion-Limited Growth

J. Kertész

5 Growth Patterns and Fronts: Fluid Flow Experiments

J. Feder, F. Boger, L. Furuberg, E. Hinrichsen,

T. Jøssang, K. J. Måløy and U. Oxaal

6.1 From Flow to Fracture and Fragmentation in Colloidal Media

1: Non-Newtonian Fingering and Visco-Elastic Fracturing

H. Van Damme and E. Lemaire

6.2 From Flow to Fracture and Fragmentation in Colloidal Media

2: Local Order and Fragmentation Geometry

H. Van Damme and M. Ben Ohoud

\section{Statistical Fracture Models}

7 Simple Stochastic Models for Material Failure

P. Meakin, G. Li, L.M. Sander, H. Yan, F. Guinea,

O. Pla and E. Louis 
P.M. Duxbury and Y. Li

9 Scaling in Fracture

H.J. Herrmann and L. de Arcangelis

\section{Rheology and Fracture}

10 Micromechanics of Brittle Deformation Processes

D. Krajcinovic

11 Fracture Mechanics and Solid Adhesion

D. Maugis

12 Damage Evolution, Instability and Fracture in Ductile Solids

A. Needleman

13 Aspects of Nonlinearity and Self organisation in Plastic Deformation E.C. Aifantis

\section{Materials and Applications}

14 Granular Media: Effects of Disorder

D. Bideau, E. Guyon and L. Oger

15 Cracking and Fracture of Concrete: Size Effect or Scaling Law?

P. Acker, P. Rossi and J-M. Torrenti

16.1 The Phenomenology of Earthquake Occurrence

L. Knopoff

16.2 The Modeling of Earthquake Occurrence

L. Knopoff 